\title{
Evaluating the effects of exercise on cognitive function in hypertensive and diabetic patients using the mental test and training system
}

\author{
Robson Bonoto Teixeira ${ }^{a}$, João Carlos Bouzas Marins ${ }^{a}$, Paulo Roberto Santos Amorim ${ }^{a}$, Israel Teoldo ${ }^{a}$, \\ Rocío Cupeiro $^{b}$, Marcelo Odilon Cabral de Andrade ${ }^{a}$, Yuri de Lucas Xavier Martins ${ }^{a}$, \\ Pollyana de Rezende Castilho ${ }^{a}$, Daniel Demétrio Magalhães ${ }^{a}$, András Palotás ${ }^{c, d}$ and Luciana Moreira Lima ${ }^{a}$ \\ ${ }^{a}$ Federal University of Viçosa, Viçosa, Minas Gerais, Brazil; ${ }^{\text {T} T e c h n i c a l ~ U n i v e r s i t y ~ o f ~ M a d r i d, ~ M a d r i d, ~ S p a i n ; ~}{ }^{\mathrm{C}}$ Kazan Federal University, \\ Kazan, Russia; ${ }^{\mathrm{d}}$ Asklepios-Med (Private Medical Practice and Research Center), Szeged, Hungary
}

ABSTRACT

Objectives: Systemic arterial hypertension (SAH) and diabetes mellitus (DM) are important risk factors for developing cognitive impairment. General life-style changes including physical training are known to reduce elevated blood pressure and sugar levels, as well as improve mental health. The objective of this study was to evaluate whether supervised physical exercise enhances the cognitive status of patients with chronic diseases.

Methods: Volunteers with SAH, DM or SAH + DM participated in either aerobic or resistance training during a period of 12 weeks. Several domains of cognitive functions were evaluated using the mental test and training system before and after the 3 months.

Results: Participants with either of these chronic diseases demonstrated significantly improved attention and concentration, but not reaction time, following the supervised exercise.

Conclusions: Structured physical training promotes several aspects of cognitive functions in diabetic and hypertensive patients.
ARTICLE HISTORY

Received 20 February 2017

Revised 20 April 2017

Accepted 23 May 2017

\section{KEYWORDS}

Cognitive function; diabetes; exercise; hypertension; mental test and training system; physical activity

\section{Introduction}

Evidence shows that elevated blood pressure levels are related to cognitive decline in both young and elderly subjects (Ninomiya et al. 2011; Guimarães et al. 2015). Moreover, the hyper-glycemic state and resistance to insulin seen in diabetes mellitus (DM) can lead to cognitive impairments caused by macro- and microvascular diseases in the brain (Qiu et al. 2014; Fava et al. 2017). Studies have also demonstrated that DM can cause alterations in the cerebral anatomical connectivity, which may potentiate motor and cognitive deficits in diabetics (Fang et al. 2017). In addition, the expression of the brain's neurotrophic factor, which acts on the maintenance of neurons, and growth and differentiation of new synapses, has been shown to be decreased in diabetics (Han et al. 2016). In a study performed during 9 years on 824 subjects aged 75 on average, who underwent detailed annual clinical evaluations, those with DM (15.4\% of the sample) had a $65 \%$ increase in the risk of developing Alzheimer's disease (AD) compared with those without DM, and had a $44 \%$ greater rate of decline in perceptual speed (Arvanitakis et al. 2004).
The mental test and training system (MTTS) is a well-established evaluation tool based on action theory, and is widely used within sports psychology (Hackfort et al. 2009), as shown by the works of Gierczuk and Ljach (2012) as well as Poliszczuk et al. (2013). However, little is known about its applicability in clinical conditions associated with an increased risk of cognitive decline, such as DM and systemic arterial hypertension $(\mathrm{SAH})$, despite the large amount of tests within MTTS that evaluate specific cognitive skills (Liu 2012; Rawlings et al. 2014).

Some studies have reported DM as a risk factor for mild cognitive decline, $A D$, vascular dementia (VD) and other types of dementia (Cheng et al. 2012), as well as associations between memory impairment and SAH. Nevertheless, the pathophysiological parameters and methodologies used in these studies are controversial (Duron and Hanon 2008). In addition, subjects with elevated blood pressure had a 3 -fold increased risk of developing VD than normotensive people, and the risk increases by 6-times when the subject presents with both SAH and DM (Posner et al. 2002). All this may significantly compromise the quality of life and

CONTACT Luciana Moreira Lima luciana.lima@ufv.br Departamento de Medicina e Enfermagem, Universidade Federal de Viçosa, Av. Peter Henry Rolfs, Viçosa, Minas Gerais, BR-36570-000, Brazil; András Palotás palotas@asklepios-med.eu E Asklepios-Med, H-6722 Szeged, Kossuth Lajos sgt. 23, Hungary 
independence in elderly people with these conditions. Moreover, these diseases are also associated with high levels of depression and anxiety and they may cause impairment in treatment compliance and in maintaining good control of the underlying disease (Teixeira et al. 2015).

Regular physical exercise can influence cognitive function by increasing brain oxygenation due to a greater blood flow and perfusion, which directly influences cognition regardless of age (Dupuy et al. 2015). However few studies investigate the use of physical activity as a strategy for reducing cognitive decline in diabetic and SAH patients, and they differ in intensity, duration and type of exercise used (Devore et al. 2009). These differences in methodologies may be the reason for the discordant results seen in the bibliography, where some studies found a small association between physical activity and cognition, as reported in observational studies in older women with DM (Swoap et al. 1994; Devore et al. 2009), whereas others reported improvements in cognitive performance after an exercise intervention. For instance, Baker et al. (2010), found an executive function enhancement in glucose-intolerant elderly subjects after 6 months of aerobic training, and Liu-Ambrose et al. (2012) described a positive impact of resistance training in elderly subjects over the functional plasticity of response inhibition processes in the cortex; an increased regional cerebral perfusion has been reported in another study ( $\mathrm{Xu}$ et al. 2014).

International guidelines state the need for including regular physical activity on DM (American Association Diabetes 2014) and SAH (Pescatello et al. 2004) treatments, especially highlighting the physiological responses, but the physical activity may also have a positive impact on cognition. Therefore, is important to know how supervised exercise training may influence cognitive aspects of DM and hypertension. This could increase the quality of life of DM and SAH patients through better concentration, attention, memory and other cognitive abilities that help in their daily lives. The aim of this study was to determine whether a 12-week programme of supervised physical exercise induces changes in the cognitive status, assessed by the MTTS, of diabetic and hypertensive patients.

\section{Materials and methods}

\section{Study design}

This is a descriptive, longitudinal-type project developed as part of the project entitled 'Global assessment of cardiovascular risk and mental state of patients assisted by the Viçosa Hiperdia Center after physical exercise programme', a partnership of the Federal University of Viçosa with Hiperdia Minas Program in Brazil. The study was conducted entirely on the premises of Hiperdia Center of the city of Viçosa, Brazil, a secondary health centre treating patients with therapy-resistant hypertension. This is a condition in which blood pressure remains above optimal levels despite the combined use of anti-hypertensive medications from at least three different classes, one being a diuretic (Daugherty et al. 2012); and/or diabetics with glycosylated haemoglobin $(\mathrm{HbA} 1 \mathrm{c})$ greater than or equal to $9 \%$.

The exercise intervention programme lasted 12 weeks and assessments were performed before (i.e. maximum graded exercise test, anthropometric measurements and MTTS) and after (i.e. MTTS) the intervention. The study was approved by the Ethics in Research Committee of the Federal University of Viçosa, Brazil (platform no. 33979214.3.0000.5153, protocol no. 832.149/2014).

\section{Participants}

All volunteers were invited to participate to the supervised physical exercise programme of the Hiperdia Center in Viçosa, MG, which registers and monitors resistant hypertensive and/or diabetic patients assisted by the Unified Health System (SUS). Hiperdia meets the resolution no. 2.606 (7 December 2010), which establishes the referral criteria for the integrated reference centres Viva Vida and Hiperdia Minas. Patient recruitment was carried out through the analysis of medical records, where patients with therapy-resistant hypertension or hypertension plus type 2DM (DM2) were selected. A phone call was made to these selected patients, where all the research procedures were clarified. If the subject agreed to participate, a first meeting in Hiperdia Center was scheduled for further information on the study procedures. Figure 1 shows the initial sample and the completers, as well as the reason for withdrawal, and the exercise groups assignment. The inclusion criteria considered were patients with DM2 and/or SAH of both genders, over 18 years old, who were not practicing routine physical exercises and/or systematized during at least 3 months. The adopted exclusion criteria were as follows: type 1 diabetics, patients with peripheral artery disease, illicit drug users, congestive heart failure, decompensated pulmonary disease, symptomatic cardiac arrhythmia, orthopaedic or rheumatological diseases that prevented the realisation of the proposed exercises, 


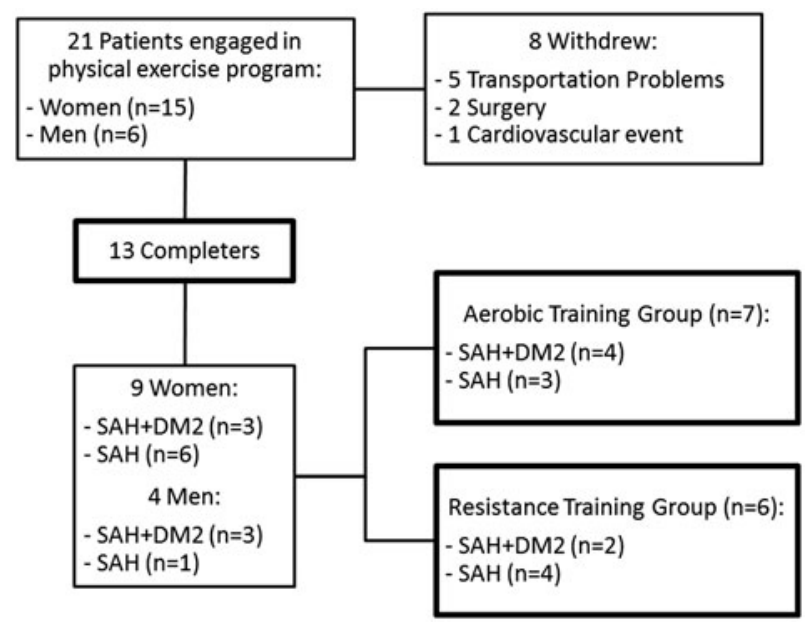

Figure 1. Initial sample size and completers, as well as the reason for withdrawal and the exercise group assignment.

symptomatic peripheral artery disease to exercise, acute cardiac ischaemia signs during the maximal graded test and symptomatic cardiac arrhythmia caused by the graded exercise test. Also, individuals using anti-depressants or anti-anxiety medications were excluded. At baseline, all participants were asked not to change their eating habits (not being prescribed any meal plan with calorie restriction). In addition, physicians were asked not to change the drug plan for any patient during the 12 weeks of training. All patients were informed about the methodology and the study's objectives and they signed the Free and Informed Consent Term.

\section{Baseline measurements}

Aiming for a possible diagnosis of cardiovascular disease, and further evaluation of symptoms consistent with arrhythmias and ischaemia induced by exercise, which are considered exclusion criteria, all patients were assessed by a graded exercise test to maximal voluntary exertion using a ramp protocol, performed by a cardiologist in the centre itself, following the rules of the Brazilian Society of Cardiology guidelines (Ghorayeb et al. 2013) with the subsequent issue of medical opinion.

The anthropometric parameters were also measured at baseline. Body weight was assessed using a Mercy scale (LC 200 model, Brazil, 2010) ranging from 1 to $200 \mathrm{~kg}$ with $50 \mathrm{~g}$ of precision; and height was measured with a stadiometer (Welmy, model R110, Brazil, 2009) ranging from 0.8 to $2.00 \mathrm{~m}$ with $1 \mathrm{~mm}$ of precision. From these data, the body mass index (BMI) was calculated, with the weight in $(\mathrm{kg})$ divided by the height squared $(m)$, with the purpose of analysing the nutritional status of the sample (Evans et al. 2012).

\section{MTTS tests}

The participants performed three tests on the MTTS with an established time of $45 \mathrm{~min}$. All tests had an adaptation period, which included a moment before performing the test itself, allowing the volunteers to familiarise themselves with the test prior measurements. The MTTS is a device consisting of an evaluation system of cognitive processes, which is the relationship between subject, environment and task. It is based on the idea that specific situations can provide important information regarding cognitive processes. The evaluation system consists of a battery of 26 tests that assess specific cognitive skills such as attention, memory, perception, knowledge and decision making. The system includes a monitor and a central processing unit, as well as one action unit, one control unit, and a peripheral equipment such as foot pedals and a peripheral perception display (Hackfort et al. 2009). This system has already been used by previous studies for assessing cognitive processes (Krzepota et al. 2015; Mańkowska et al. 2015).

The tests performed on the equipment were: cognitrone (COG, version S4) that measures attention and concentration; the determination test (DT, version S1) which assesses the participants' reaction time; and the visual pursuit test (LVT, version S3) to evaluate selective attention. These three tests were performed within the same assessment session, in the cited order, at the Center of Research and Studies in Soccer located in the Department of Physical Education of the Federal University of Viçosa, Brazil. The tests were applied by a physical education professional with high experience in handling the equipment.

The cognitrone test (hereafter referred to as Attention and Concentration test) assesses attention and concentration by recognising one figure from among a set of figures presented, as quickly as possible. The test requires a reaction only if the figure given is within the set presented. The variables recorded in this test were number of correct reactions, number of incorrect reactions (i.e. the figure selected was not correct) and the number of omitted reactions (i.e. the participant did not select any figure within the given time) (Schuhfried 2011a).

The DT assesses the volunteers' reaction time, testing their reactive stress tolerance and the reaction time associated. During the test, the participant has to react to visual and acoustic stimuli pressing specific buttons for each stimulus. The variables analysed were: number of correct reactions, that is the main variable of this test, and represents the total number of appropriate reactions to the stimuli; the number of 
incorrect reactions, which is the total number of inadequate reactions to the stimuli; and the median reaction time, which defines the interval between the presentation of the stimulus and the pressing of the appropriate button (Schuhfried 2011b).

For the evaluation of selective attention, the visual pursuit test was used, whereby subjects have to identify as quickly as possible, the end of a given line that is part of a maze composed of more lines. The difficulty increases during the session. The variables recorded are the number of correct reactions, the time spent to complete the test (higher score is given to less time needed to finish), and the median time of correct reactions, that is the median of the time intervals spent in each correct reaction (Biehl 2011). The initial screens of the tests used are shown in Figure 2.

As MTTS does not have an assessment scale, results obtained at baseline and after the intervention were compared with each other.

\section{Exercise programme}

The volunteers who fulfilled the inclusion criteria were randomly allocated in one of the two exercise groups: aerobic or resistance training (Figure 1), both of them lasting 12 weeks and with a training frequency of three times per week. All training sessions were supervised by graduates in physical education. A minimum of $90 \%$ of adherence to the programme was required to validate the results.

The exercise programme followed the recommendations for exercising in diabetic population of the American College of Sport Medicine (Colberg et al. 2010) and the American Diabetes Association (American Association Diabetes 2014), and the recommendations for hypertensive population of the American College of Sports Medicine (Pescatello et al. 2004).

Seeking an appropriate physiological and motor adaptation, the volume of both exercise programmes (i.e. aerobic or resistance training) increased progressively during the first weeks, so that the main part of the session lasted 20 min within the first week, 30 min in the second week and $40 \mathrm{~min}$ in the following weeks.

All training sessions started with a warm-up on a cycle ergometer for $10 \mathrm{~min}$ at $50 \%$ of the maximum heart rate, estimated by the Tanaka et al. equation MHR $=208-(0.7 \times$ age $)$ (Tanaka et al. 2001), and ended with a 10-min cool down of active and passive
(A)

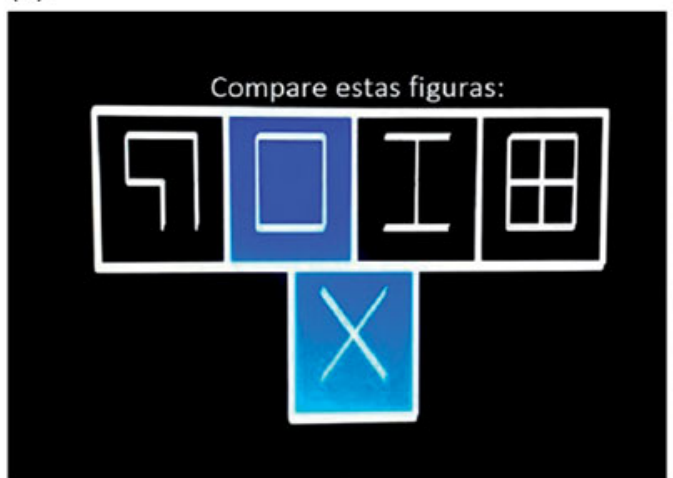

(C)
(B)

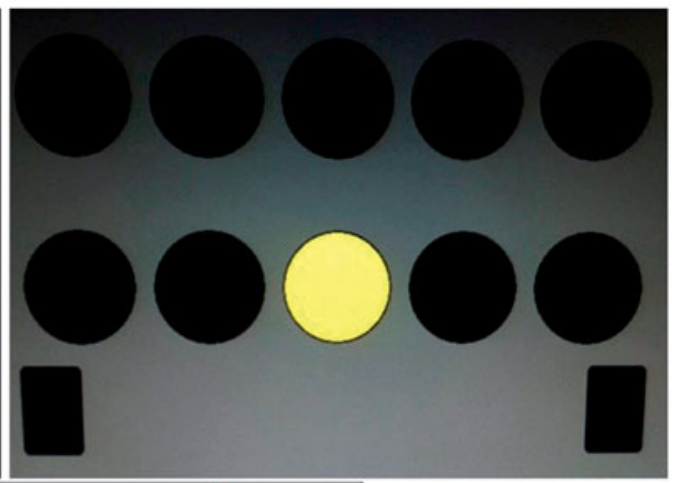

$\downarrow$

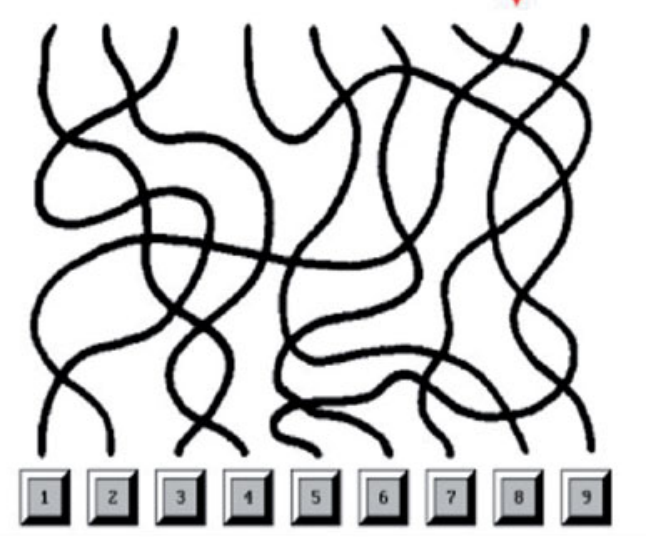

Figure 2. (A) Initial screen for the cognitive test or Attention and Concentration test; (B) initial screen for the determination test or reaction time test; $(C)$ visual pursuit test or selective attention test. 
stretching exercises of the major muscle groups (quadriceps, hamstrings, large dorsal, chest, back). Therefore, from the third week onward, the average time to complete each exercise session was around 50-60 min. Participants of the resistance group followed a sequence of ten exercises with $15 \mathrm{~s}$ of recovery between them: seated cable row, squats, dumbbells bench press, knee extension with ankle weights, dumbbell shoulder press, dumbbell biceps curl, standing knee flexion with ankle weights, standing calf raises, cable rope overhead triceps extension and abdominal crunch. The circuit method with an interval of $15 \mathrm{~s}$ between exercises was used in the first 2 weeks in order to adapt the neural, joint and muscle systems, and the volunteers performed two sets of 15 repetitions. After this period, the training consisted of three sets of 12 repetitions. The exercises were performed in a controlled fashion, with moderate speed and similar duration between the concentric and eccentric phases. Regarding the aerobic group, participants performed 20,30 or $40 \mathrm{~min}$ of aerobic exercise (depending on the progression phase), in bouts of $10-20 \mathrm{~min}$ without a rest between them, using one of the following devices (self-selected by the participant): treadmill, bike, elliptical or upper-body cycle ergometer.

The intensity for both programmes was prescribed individually, respecting the limitations and potential of each patient. The intensity of the resistance exercise programme was controlled using the perceived effort by means of the 6-20 Borg scale (Borg 1982). The participants had to exercise at an intensity of $11-12$, representing a moderate effort (Kesaniemi et al. 2001). Even with the load adjustments, the participants' effort perception has always remained in the range from 11 to 12. As a large part of the sample was illiterate, or with low educational level, at the time of Borg's scale application the researcher individually read each score of the scale and the intensity of the exercise that this score represented, and the patient had to indicate his perception of effort. The Borg scale had already been used in illiterate patients, as in the study by Martinez et al. (2000). Moreover the prescription and training load control in strength exercises was made from the perceived exertion due to low levels of physical fitness and motor coordination and to treat patients with high cardiovascular risk. Load testing or maximum repetitions would entail a non-consistent effort with the health condition of the participant, which would increase the risk of adverse events. Therefore, for safety, the training loads were adjusted to the extent that the physical fitness and motor skills of the participants improved, always taking into account the perceived exertion as moderate.
For the aerobic programme, the intensity was set at $60 \%$ of the maximal heart frequency estimated by the Tanaka equation (Tanaka et al. 2001), except for those taking $\beta$-blockers. If the volunteer was on these medications, exercise intensity was controlled by the Borg scale, using the values 11-12, which represent a moderate effort, besides having a strong association with the average percentage of cardiac frequency (David and Julen 2015). Heart rate was controlled using the heart rate monitors integrated in the ergometers, for the sole purpose of controlling the exercise intensity.

In order to avoid any health event, measurements of blood pressure were performed in all participants before each exercise session, as well as $30 \mathrm{~min}$ after its beginning and $20 \mathrm{~min}$ after the end of each session. At the same time points, measurements of blood glucose were conducted in diabetic participants. The glucometer Roche Accu Chek Performa ${ }^{\circledR}$ (Mannheim, Germany, 2009) was used to measure the glucose, while a stethoscope and aneroid sphygmomanometer (Premium, Wenzhou Instrument Co., China, 2014) were used for the blood pressure measurements.

\section{Statistical analysis}

Standard statistical methods were used for the calculation of means and standard deviation ( \pm SD). The Saphiro-Wilk test was used to analyse the normality of the variables. Afterwards, pre-post comparisons were made by means of a paired $t$-test for variables with normal distribution, and by means of the Wilcoxon test for those that were not normally distributed. Fisher's exact test was used for frequency comparisons. On the other hand, regarding the MTTS tests, the Pearson correlation was used to established associations between variables: between 'correct reactions' and 'incorrect reactions' within the Attention and Concentration test; between 'correct reactions' and 'median reaction time' within the Reaction Time test; and 'correct reactions' and 'median time of correct reactions' in the Selective Attention test. Significance was set at $\alpha=0.05$. All analyses were performed using SPSS v.20.0 software (SPSS Inc., Chicago, IL).

\section{Results}

Table 1 shows the sample characteristics. Considering account values of BMI $\geq 25 \mathrm{~kg} / \mathrm{m}^{2}$ (overweight) and $\geq 30 \mathrm{~kg} / \mathrm{m}^{2}$ (obesity) (Evans et al. 2012), 69\% of the women and $92 \%$ of the total sample showed excess weight body, whereas no significant differences were observed between groups for age and education level. 
Table 2 shows the results for the MTTS tests for hypertensive and for diabetics plus hypertensive participants at baseline and after the exercise intervention. There was no change on reaction time levels and selective attention after the programme. However, in the Attention and Concentration test, the group of diabetics plus hypertensive patients showed a significant increase on 'correct reactions', as well as on 'incorrect reactions', reflecting an augmented response to the stimuli presented after the intervention. For this group the 'omitted reactions' variable also improved, demonstrating a decrease in the cases of non-identification of two similar figures. In contrast, in the hypertensive group this variable significantly decreased, exhibiting a higher number of times the participants did not identify the correct figure.

When comparing exercise groups, there were no differences between baseline and post-intervention values in any of the tests performed for either group (Figure 3).

Taking in account the complete sample, a negative correlation was observed between 'correct reactions'

Table 1. Sample characterisation.

\begin{tabular}{lcccc}
\hline & $\begin{array}{c}\text { Total } \\
(n=13)\end{array}$ & $\begin{array}{c}\text { SAH } \\
(n=7)\end{array}$ & $\begin{array}{c}\text { SAH + DM2 } \\
(n=6)\end{array}$ & $P$ \\
\hline Age (years) & $55 \pm 12$ & $60 \pm 9$ & $49 \pm 13$ & $.218 \neq$ \\
Gender (male/female) & $4 / 9$ & $1 / 6$ & $3 / 3$ & - \\
BMl & & & 0 & - \\
Underweight & 0 & 0 & $1(17 \%)$ & - \\
Normal weight & $1(8 \%)$ & 0 & $2(33 \%)$ & $.592 \#$ \\
Overweight & $6(46 \%)$ & $4(57 \%)$ & $3(50 \%)$ & $.616 \#$ \\
$\begin{array}{l}\text { Obesity } \\
\text { Education (years) }\end{array}$ & $6(46 \%)$ & $3(43 \%)$ & & \\
Illiterate & $5(38 \%)$ & $3(42 \%)$ & $2(33 \%)$ & $.587 \#$ \\
$0-3$ & $4(31 \%)$ & $3(43 \%)$ & $1(17 \%)$ & $.559 \#$ \\
$4-8$ & $3(23 \%)$ & $1(14 \%)$ & $2(33 \%)$ & $.559 \#$ \\
8 or more & $1(8 \%)$ & 0 & $1(17 \%)$ & - \\
\hline SAH & & &
\end{tabular}

SAH: systemic arterial hypertension; SAH + DM2; systemic arterial hypertension plus type 2 diabetes mellitus; BMI: body mass index.

$\neq$ Student's $t$-test, data presented as mean \pm SD. $\neq$ Fischer's exact test, data presented as number of participants and percentage. and 'omitted reactions' $(r=-0.999 ; P=.001)$ in the cognitrone test. This correlation means that as the higher the number of 'correct reactions' appears, a lower number of 'omitted reactions' was registered. Another strong negative correlation was observed within the DT between 'correct reactions' and 'median reaction time' $(r=-0.904 ; P=.001)$, which shows that, as the number of correct reactions was greater; the time interval between stimulus presentation and correct reaction was lower.

Finally, in the visual search test, a negative correlation was found between 'correct reactions' and 'median time of correct reactions' $(r=-0.689$; $P=.009$ ). That is, the greater the number of 'correct reactions' the lower the time needed for these correct reactions.

\section{Discussion}

As far as the authors know, this is the first study to assess the cognitive parameters in diabetic and hypertensive patients using the MTTS equipment. The main finding was the increase in the number of 'correct reactions' and 'incorrect reactions', as well as the decrease in the number of 'omitted reactions', of a test assessing attention and concentration, after a 12week resistance or aerobic exercise intervention in patients with SAH plus DM2. Since there was an increase in the number of 'correct reactions', it was verified that the participants were found to be more attentive and reacting more easily to the test stimuli after 12 weeks of supervised exercise. In addition, there was a significant drop in the 'omitted reactions' variable, suggesting that the participants reacted to the actions imposed by the test, thus evidencing higher levels of attention and concentration, since omission is also considered as an error (Schuhfried

Table 2. Results for the mental test and training system (MTTS) at baseline and after the 12-week exercise intervention in the diabetic and hypertensive groups.

\begin{tabular}{|c|c|c|c|c|c|c|}
\hline & \multicolumn{3}{|c|}{$\mathrm{SAH}(n=7)$} & \multicolumn{3}{|c|}{$\mathrm{SAH}+\mathrm{DM} 2(n=6)$} \\
\hline & Baseline & Post-intervention & $P$ & Baseline & Post-intervention & $P$ \\
\hline \multicolumn{7}{|c|}{ Cognitrone (attention and concentration) test } \\
\hline Correct reactions & $34 \pm 15$ & $29 \pm 15$ & $.621^{\neq}$ & $22 \pm 11$ & $51 \pm 15$ & $.013^{\neq}$ \\
\hline Incorrect reactions & $38 \pm 22$ & $32 \pm 18$ & $.609^{\neq}$ & $22 \pm 9$ & $50 \pm 28$ & $.028 \#$ \\
\hline Omitted reactions & $37 \pm 11$ & $51 \pm 15$ & $.031^{\neq}$ & $59 \pm 11$ & $29 \pm 15$ & $.013^{\neq}$ \\
\hline \multicolumn{7}{|l|}{ Determination (reaction time) test } \\
\hline Correct reactions & $107 \pm 45$ & $90 \pm 60$ & $.595^{f}$ & $104 \pm 58$ & $150 \pm 54$ & $.153^{\neq}$ \\
\hline Incorrect reactions & $18 \pm 14$ & $17 \pm 11$ & $.871^{\neq}$ & $9 \pm 6$ & $24 \pm 15$ & $.096^{\neq}$ \\
\hline Median reaction time $(\mathrm{s})$ & $1,3 \pm 0,2$ & $1,4 \pm 0,3$ & $.367^{\neq}$ & $1,5 \pm 0,6$ & $1,2 \pm 0,2$ & $.258^{\neq}$ \\
\hline \multicolumn{7}{|l|}{ Visual pursuit (selective attention) test } \\
\hline Correct reactions & $15 \pm 2$ & $11 \pm 5$ & $.176^{\neq}$ & $10 \pm 7$ & $16 \pm 1$ & $.116^{\neq}$ \\
\hline Median time of correct reactions (s) & $6 \pm 2$ & $9 \pm 7$ & $.413^{f}$ & $6 \pm 2$ & $6 \pm 2$ & $.959^{\neq}$ \\
\hline Time for complete the test (s) & $126 \pm 47$ & $198 \pm 150$ & $.331^{\neq}$ & $102 \pm 21$ & $111 \pm 48$ & $.635^{\neq}$ \\
\hline
\end{tabular}

Data are presented as mean \pm SD. SAH: systemic arterial hypertension; SAH + DM2: systemic arterial hypertension plus type 2 diabetes mellitus.

$\neq$ Student's $t$-test; \#non-parametric test. 


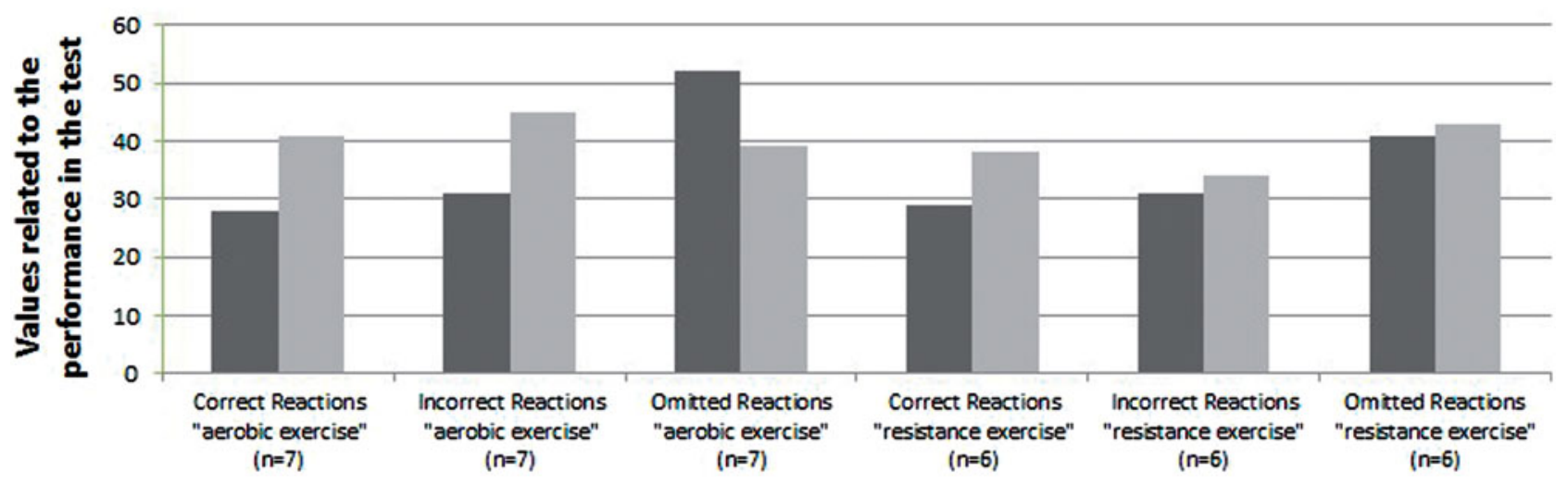

a Pre-Workout $=$ Post-Workout

Figure 3. Comparison of variables in Attention and Concentration test of aerobic and resistance training groups before and at the end of the 12 weeks of supervised training.

2011a). In contrast, the number of 'incorrect reactions' also increased after the intervention period. This result can be explained by the greater readiness for action of the study participants, where even making mistakes in greater quantity, the participants were able to perceive environmental situations in the attempt of better decision making (Posner et al. 2002). In this way, these results demonstrate improvements in attention and concentration, both of them key aspects for DM and SAH people.

A possible explanation for improvement in the DM and SAH groups is that the participants in this group were less focussed and attentive before the intervention than the SAH group, so it was inferred that the patients with DM and SAH had a higher propensity for improvement. Another possible explanation would be that the association between diabetes and hypertension could lead to greater cognitive impairment for the patient than the existence of only one disease (Petrova et al. 2010). However further studies should be conducted to answer this question. Advances in attention and concentration levels is important for diabetic and hypertensive subjects because they usually need take several medications at different hours and have to be careful with their food intake. Moreover, attention and concentration can also improve their socialisation with relatives and friends, improving their quality of life. The lack of differences found between aerobic or resistance exercise implies that both types of training can be introduced as good strategies for the non-pharmacological treatment of DM and SAH.

The BMI of the sample was above the recommended values (Evans et al. 2012), with 92\% of the participants being overweight or obese. A growing body of research indicates that obesity in middle age is an important predictor of cognitive decline in old age (Odegaard and Chawla 2013). Obesity is a white adipose tissue accumulation, promoting the process of systemic inflammation (Odegaard and Chawla 2013). This may contribute to cognitive decline and dementia due to the release of several pro-inflammatory cytokines, such as IL-1 $\beta$ and IL- 6 , which are known to disrupt neural circuits involved in cognition and memory (Nguyen et al. 2014). Prickett et al (2015), as well as Bischof and Park (2015), showed in their respective reviews that there is strong evidence for obesity leading to cognitive deficit, especially in the elderly. Nevertheless, the authors point out that there are many methodological problems with the reviewed studies. Therefore, more studies are needed for evidencing obesity as a risk factor for cognitive impairment.

The fact that most of the sample were illiterate and had no access to electronic devices could have influenced their performance during the MTTS tests, since the tests are carried out using modern and technic equipment. However, it is believed that the familiarisation phase before the test helped to minimise this influence, since differences were found in the profile of satisfactory results in the Attention and Concentration test. Most tests for cognitive analysis require reading and writing skills, such as the Alzheimer's Disease Assessment Scale - cognitive subscale (ADAS-COG) or the Consortium to establish a registry for Alzheimer's disease (CERAD), and this could be a limitation in the analysis of an illiterate population. Therefore, the development of measurement tools for the evaluation of cognition is essential regardless of the educational level, such as literacyindependent cognitive assessment, which is based on oral and visual responses. This reduces the number of false cognitive impairment diagnoses (Shim et al. 2015). It is important to note that the tests chosen for the study were carefully selected so that the patient 
does not need necessarily need to be literate. Thus, the educational level of the sample probably was not a confounding factor during the evaluation.

Significant improvements were found in the variable score of 'omitted reactions' in the Attention and Concentration test after exercise intervention. This implies that, after the exercise programme, the participants did not press any key when they did not identify any figure as correct, because they were more focussed and had higher attention. Several studies show that physical exercise improves cognitive ability. Smith et al. (2010) reviewed 29 works, with a total of 2049 participants, and found that subjects who received aerobic training obtained slight improvement in attention levels and processing speed. Furthermore, it has been reported that cognitive attention and memory improvements are maintained 9 months after a cardiac rehabilitation programme, raising the possibility that supervised exercise reduces the risk of dementia in patients with cardiovascular disease or attenuate cognitive decline (Alosco et al. 2014). A study conducted by Strassnig et al. (2015) with 12 psychiatric patients (schizophrenic and bipolar) who were overweight, showed a significant improvement in musculoskeletal and cognitive capacities, as well as in psychiatric symptoms. In agreement with these findings, Vidoni et al. (2015), comparing different training volumes (i.e. 75,150 or $225 \mathrm{~min}$ of walking) in older adults, observed improved cognitive function in all the exercise groups compared with the control group without training, and that the greater the volume training the better the physical benefits achieved. However, it is necessary to highlight that none of these studies used the MTTS as an evaluation tool. In addition, it seems that it is still unclear what kind of exercise, aerobic or resistance presents the greatest benefits for mental status.

The results indicate that the selected exercise programmes improve the reaction of diabetic and hypertensive participants, since they have reacted more often during the Attention and Concentration test because 'correct reactions' and 'incorrect reactions' have increased. The negative correlation found between 'correct reactions' and 'omitted reactions' in the hypertensive plus diabetic patients, show that the exercise programme causes the participants to perceive more stimulus and, consequently, to achieve better results. The negative correlations observed between 'correct reactions' and 'median reaction time' within the reaction time test, and between 'correct reactions' and 'median time of correct reactions', indicate a shorter interval of time needed to obtain correct responses after the exercise intervention. Despite the absence of differences between the reference and post-intervention measures in the selective attention and reaction time tests, a greater reaction of the participants was observed, that is, they spent less time providing the correct response after the 12 weeks of training. In addition, increases of $31 \%$ and $38 \%$ were recorded in the variables 'correct reactions' of these tests (reaction time and selective attention, respectively), showing what appears to be the beginning of a positive evolution and representing a considerable clinical improvement. Maybe the duration of the intervention (12 weeks) partially determined this lack of significant differences that have been reported in previous works. For instance, Liu-Ambrose et al. (2012) observed improvements in the levels of selective attention and conflict resolution, using the Stroop test, in 65-75-year-old women performing resistance training during 12 months. On the same lines, Baker et al. (2010) demonstrated that 6 months of aerobic training were enough to improve selective attention tasks in elderly men, whereas their control group without exercise training did not improve. Perhaps, the inclusion of activities, such as dance and simple games, would require more complex cognitive processes, and would provide more benefits to the participants (Teixeira et al. 2015).

It is assumed that this is the first study using MTTS tests to evaluate the benefits of supervised exercise on the cognitive function of patients with T2DM and SAH. MTTS equipment was used in this population because these subjects have a higher risk of cognitive decline. It is believed that equipment like this should be tested and used not only by athletes, who are a minority of the population, but also by 'ordinary' people, helping in their integral treatment. Besides, the non-existence of a normal pattern makes it difficult to compare the results of this study with others from healthy populations. This reinforces the importance of the results obtained in this study, since they can be used to create normative standards, facilitating the comparison between different studies and populations. However, further studies are needed to confirm the MTTS as an assessment tool for therapeutic interventions in patients with DM2 and SAH.

\section{Study limitations}

The main limitation of the study was the sample size. However, the special features of the sample, and the lack of work on this focus, make this research useful for future studies to investigate larger samples to confirm the importance of prescriptive exercise on the cognitive aspects for DM and SAH subjects. Another limitation may have been the duration of the exercise 
programme. It is possible that an intervention of 6 months or more would have produced greater improvements. Therefore, a longer exercise intervention is proposed for future work.

\section{Conclusions}

The results suggest a positive influence of supervised exercise on cognitive processes in patients with DM plus SAH. Significant improvements in attention and concentration levels were observed after 12 weeks of supervised resistance or aerobic exercise. In contrast, there were no significant differences in the reaction time test and selective attention test responses, although improvements were observed in some variables, indicating a clinical improvement. As MTTS is not used routinely to evaluate the cognitive state of patients with DM and $\mathrm{SAH}$, prospective studies with follow-up involving a larger number of individuals are essential to clarify, with reliability, the use of MTTS for the assessment of cognitive state in patients with these diseases.

\section{Acknowledgments}

This study was sponsored by the Fundação de Amparo à Pesquisa de Minas Gerais (FAPEMIG), Brazil. JCBM is funded by a productivity grant of the Conselho Nacional de Desenvolvimento Científico e Tecnológico (CNPq), Brazil. RBT owns a pre-doctoral grant of the Coordenação de Aperfeiçoamento de Pessoal de Nível Superior (CAPES), Brazil. PRC and DDM are funded by undergraduate grants from the CNPq and CAPES, respectively.

\section{Statement of interest}

None to declare.

\section{Funding}

This work was supported by Fundação de Amparo à Pesquisa do Estado de Minas Gerais (FAPEMIG), Brazil, APQ02612-15.

\section{References}

Alosco ML, Spitznagel MB, Cohen R, Sweet LH, Josephson R, Hughes J, Gunstad J. 2014. Decreases in body mass index after cardiac rehabilitation predict improved cognitive function in older adults with heart failure. J Am Geriatr Soc. 62:2215-2216.

American Association Diabetes. 2014. Standards of medical care in diabetes-2014. Diabetes Care. 37:14-80.

Arvanitakis Z, Wilson RS, Bienias JL, Evans DA, Bennett DA. 2004. Diabetes mellitus and risk of alzheimer disease and decline in cognitive function. Arch Neurol. 61:661-666.
Baker LD, Frank LL, Foster-Schubert K, Green PS, Wilkinson CW, McTiernan A, Cholerton BA, Plymate SR, Fishel MA, Watson GS, et al. 2010. Aerobic exercise improves cognition for older adults with glucose intolerance, a risk factor for Alzheimer's disease. Int. J Alzheimers Dis. 22:569-579.

Biehl B. 2011. Visual Pursuit Test. In: Schuhfried GMBH, editor. 1st ed. Modling: Vienna Test System, 68.

Bischof GN, Park DC. 2015. Obesity and aging: consequences for cognition, brain structure, and brain function. Psychosom Med. 77:697-709.

Borg GA. 1982. Psychophysical bases of perceived exertion. Med Sci Sports Exerc. 14:377-381.

Cheng G, Huang C, Deng H, Wang H. 2012. Diabetes as a risk factor for dementia and mild cognitive impairment: a metaanalysis of longitudinal studies. Intern Med J. 42:484-491.

Colberg SR, Sigal RJ, Fernhall B, Regensteiner JG, Blissmer BJ, Rubin RR, Chasan-Taber L, Albright AL, Braun B. 2010. Exercise and type 2 diabetes the American College of Sports Medicine and the American Diabetes Association: joint position statement. Diabetes Care. 33:147-167.

Daugherty SL, Powers JD, Magid DJ, Tavel HM, Masoudi FA, Margolis KL, O'Connor PJ, Selby JV, Ho PM. 2012. Incidence and prognosis of resistant hypertension in hypertensive patients. Circulation. 125:1635-1642.

David C, Julen C. 2015. The relationship between intensity indicators in small-sided soccer games. J Hum Kinet. 46:119-128.

Devore EE, Kang JH, Okereke O, Grodstein F. 2009. Physical activity levels and cognition in women with type 2 diabetes. Am J Epidemiol. 170:1040-1047.

Dupuy O, Gauthier CJ, Fraser SA, Desjardins-Crepeau L, Desjardins M, Mekary S, Lesage F, Hoge RD, Pouliot $P$, Bherer L. 2015. Higher levels of cardiovascular fitness are associated with better executive function and prefrontal oxygenation in younger and older women. Front Hum Neurosci. 9:66.

Duron E, Hanon O. 2008. Hypertension, cognitive decline and dementia. Arch Cardiovasc Dis. 101:181-189.

Evans PD, Mclntyre NJ, Fluck RJ, Mclntyre CW, Taal MW. 2012. Anthropomorphic measurements that include central fat distribution are more closely related with key risk factors than BMI in CKD stage 3. PloS One. 7:34699.

Fang P, An J, Tan X, Zeng LL, Shen H, Qiu S, Hu D. 2017. Changes in the cerebellar and cerebro-cerebellar circuit in type 2 diabetes. Brain Res Bull. 11:95-100.

Fava A, Colica C, Plastino M, Messina D, Cristiano D, Opipari C, Vaccaro A, Gorgone G, Bosco F, Fratto A,D, Bartolo M, Bosco D. 2017. Cognitive impairment is correlated with insulin resistance degree: the 'PA-NICO-study'. Metabolic Brain Dis. 32:799-810.

Ghorayeb N, Costa RV, Castro I, Daher DJ, Oliveira Filho JA, Oliveira MA. 2013. Guidelines on exercise and sports cardiology from the Brazilian Society of Cardiology and the Brazilian Society of Sports Medicine. Arq Bras Cardiol. 100 (1 Suppl 2):1-41.

Gierczuk D, Ljach W. 2012. Evaluating the coordination of motor abilities in Greco-Roman wrestlers by computer testing. Hum Mov Sci. 13:323-329.

Guimarães FC, dos Santos Amorim PR, Dos Reis FF, Bonoto RT, De Oliveira WC, da Silva Moura TA, Lima LM. 2015. Physical activity and better medication compliance improve mini-mental state examination scores in the elderly. Dement Geriatr Cogn Disord. 39:25-31. 
Hackfort D, Kilgallen C, Hao L. 2009. The Action TheoryBased Mental Test and Training System (MTTS). In: Hung TM, Lidor R, Hackfort D, editors. Psychology of Sport Excellence: International Perspectives on Sport and Exercise Psychology. Morgantown: Fitness Information Technology; p. 15-24.

Han H, Wu LM, Han MX, Yang WM, Wang YX, Fang ZH. 2016. Diabetes impairs spatial learning and memory and hippocampal neurogenesis via BDNF in rats with transient global ischemia. Brain Res Bull. 124:269-277.

Kesaniemi Y, Danforth E, Jr Jensen MD, Kopelman PG, Lefèbvre $P$, Reeder BA. 2001. Dose-response issues concerning physical activity and health: an evidence-based symposium. Med Sci Sports. 33:351.

Krzepota J, Zwierko T, Puchalska-Niedbał L, Markiewicz M, Florkiewicz B, Lubiński W. 2015. The efficiency of a visual skills training program on visual search performance. J Hum Kinet. 46:231-240.

Liu-Ambrose T, Nagamatsu LS, Voss MW, Khan KM, Handy TC. 2012. Resistance training and functional plasticity of the aging brain: a 12-month randomized controlled trial. Neurobiol Aging. 33:1690-1698.

Liu H. 2012. Elaboration and Application of a Mental Test and Training System (MTTS) Tool in the Frame of Actiontheory-based Mental Assessment and Training Approach: München, Univ. der Bundeswehr, Diss.

Mańkowska M, Poliszczuk T, Poliszczuk D, Johne M. 2015. Visual perception and its effect on reaction time and timemovement anticipation in elite female basketball players. Pol J Sport Tourism. 22:3-8.

Martinez JA, Straccia L, Sobrani E, Silva GA, Vianna ÉO, Terra FJ. 2000. Dyspnea scales in the assessment of illiterate patients with chronic obstructive pulmonary disease. Am J Med Sci. 320:240-243.

Nguyen JC, Killcross AS, Jenkins TA. 2014. Obesity and cognitive decline: role of inflammation and vascular changes. Front Neurosci. 8:375.

Ninomiya T, Ohara T, Hirakawa Y, Yoshida D, Doi Y, Hata J, Kanba S, Iwaki T, Kiyohara Y. 2011. Midlife and late-life blood pressure and dementia in Japanese elderly: the Hisayama study. Hypertension. 58:22-28.

Odegaard Jl, Chawla A. 2013. Pleiotropic actions of insulin resistance and inflammation in metabolic homeostasis. Science. 339:172-177.

Pescatello LS, Franklin BA, Fagard R, Farquhar WB, Kelley GA, Ray CA. 2004. American College of Sports Medicine position stand. Exercise and hypertension. Med Sci Sports Exerc. 36:533-553.

Petrova M, Prokopenko S, Pronina E, Mozheyko E. 2010. Diabetes type 2, hypertension and cognitive dysfunction in middle age women. J Neurol Sci. 299:39-41.

Poliszczuk T, Poliszczuk D, Da, browska-Perzyna A, Johne M. 2013. Asymmetry of complex reaction time in female épée fencers of different sports classes. Pol J Sport Tourism. 20:25-29.

Posner HB, Tang MX, Luchsinger J, Lantigua R, Stern $\mathrm{Y}$, Mayeux R. 2002. The relationship of hypertension in the elderly to $A D$, vascular dementia, and cognitive function. Neurology. 58:1175-1181.
Prickett C, Brennan L, Stolwyk R. 2015. Examining the relationship between obesity and cognitive function: a systematic literature review. Obes Res Clin Pract. 9:93-113.

Qiu C, Sigurdsson S, Zhang Q, Jonsdottir MK, Kjartansson O, Eiriksdottir G, Garcia ME, Harris TB, van Buchem MA, Gudnason V, et al. 2014. Diabetes, markers of brain pathology and cognitive function: the Age, Gene/Environment Susceptibility-Reykjavik Study. Ann Neurol. 75:138-146.

Rawlings AM, Sharret AR, Schneider A, Coresh J, Albert M, Couper D, Griswold M, Gottesman RF, Wagenknecht LE, Windham BG, et al. 2014. Diabetes in midlife and cognitive change over 20 years: the atherosclerosis risk. In communities neurocognitive study. Ann Inter Med. 161:785-793.

Schuhfried G. 2011a. Cognitrone Test. In: Schuhfried GMBH, editor. 1st ed. Modling: Vienna Test System, 57.

Schuhfried G. 2011b. Determination Test. In: Schuhfried $\mathrm{GMBH}$, editor. 1st ed. Modling: Vienna Test System, 61.

Shim Y, Ryu HJ, Lee DW, Lee JY, Jeong JH, Choi SH, Han SH, Ryu SH. 2015. Literacy independent cognitive assessment: assessing mild cognitive impairment in older adults with low literacy skills. Psychiatry Investig. 12:341-348.

Smith PJ, Blumenthal JA, Hoffman BM, Cooper H, Strauman TA, Welsh-Bohmer K, Browndyke JN, Sherwood A. 2010. Aerobic exercise and neurocognitive performance: a metaanalytic review of randomized controlled trials. Psychosom Med. 72:239-252.

Strassnig MT, Signorile JF, Potiaumpai M, Romero MA, Gonzalez C, Czaja S, Harvey PD. 2015. High velocity circuit resistance training improves cognition, psychiatric symptoms and neuromuscular performance in overweight outpatients with severe mental illness. Psychiatry Res. 229:295-301.

Swoap R, Norvell N, Graves J, Pollock M. 1994. High versus moderate intensity aerobic exercise in older adults: psychological and physiological effects. J Aging Phys Act. 2:293-303.

Tanaka H, Monahan KD, Seals DR. 2001. Age-predicted maximal heart rate revisited. J Am Coll Cardiol. 37:153-156.

Teixeira RB, Marins JC, de S, Junior AR, de Carvalho CJ, Lade CG, Rizvanov AA, Kiyasov AP, Mukhamedyarov MA, Palotás A, Lima LM. 2015. Psychological and cognitive profile of hypertensive and diabetic patients. J Nerv Ment Dis. 203:781-785.

Teixeira RB, Marins JC, de S, Junior AR, de Carvalho CJ, Moura TAS, Lade CG, Rizvanov AA, Kiyasov AP, Mukhamedyarov MA, Zefirov $A L$, et al. 2015. Improved cognitive, affective and anxiety measures in patients with chronic systemic disorders following structured physical activity. Diab Vasc Dis Res. 12:445-454.

Vidoni ED, Johnson DK, Morris JK, Van Sciver A, Greer CS, Billinger SA, Donnelly JE, Burns JM. 2015. Dose-response of aerobic exercise on cognition: a community-based, pilot randomized controlled trial. PLoS One. 10:e0131647.

Xu X, Jerskey BA, Cote DM, Walsh EG, Hassenstab JJ, Ladino ME, Clark US, Labbe DR, Gunstad JJ, Poppas A, et al. 2014. Cerebrovascular perfusion among older adults is moderated by strength training and gender. Neurosci Lett. 560:26-30. 